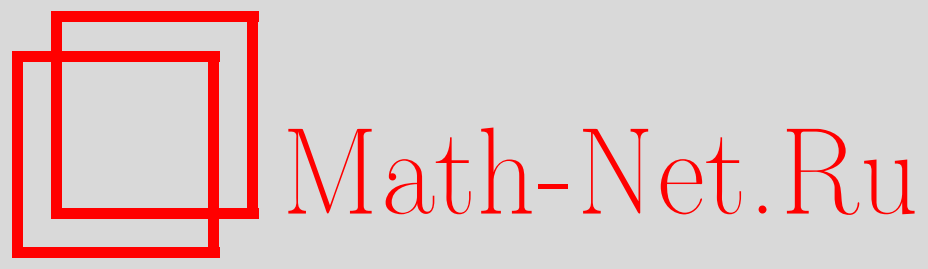

Г. М. Губреев, Ю. Д. Латушкин, Возмущения сильно непрерывных полугрупп операторов и матричные веса Макенхаупта, Функи. анализ и его прил., 2008, том 42, выпуск 3, 85-89

DOI: https://doi.org/10.4213/faa2916

Использование Общероссийского математического портала MathNet.Ru подразумевает, что вы прочитали и согласны с пользовательским соглашением http://www . mathnet.ru/rus/agreement

Параметры загрузки:

IP: 34.239 .49 .27

26 апреля 2023 г., 07:57:14

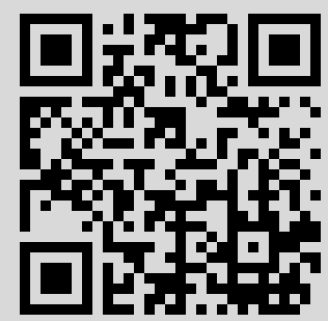


УДК 517.98

\title{
Возмущения сильно непрерывных полугрупп операторов и матричные веса Макенхаупта*
}

\author{
(c) 2008. Г. М. ГУБРЕЕВ, Ю. Д. ЛАТУШКИН
}

К столетию М. Г. Крейна

В этой работе функциональные модели несамосопряженных операторов и техника матричных весов Макенхаупта применяются к теории однопараметрических полугрупп операторов в гильбертовых пространствах.

1. $\boldsymbol{w}$-возмущения линейных операторов. Пусть $A, A_{0}$ - линейные неограниченные плотно заданные и непрерывно обратимые операторы в сепарабельном гильбертовом пространстве $\mathfrak{H}$, причем имеет место равенство

$$
A^{-1} h=A_{0}^{-1} h+\sum_{k=1}^{n}\left(h, f_{k}\right) g_{k}, \quad h \in \mathfrak{H},
$$

где $f_{k}, g_{k} \in \mathfrak{H}(1 \leqslant k \leqslant n)$, а скобки обозначают скалярное произведение в $\mathfrak{H}$. Будем предполагать, что оператор $A_{0}$ принадлежит классу $\Sigma^{(\exp )}$, т. е. [1];

а) $A_{0}$ не имеет спектра и генерирует $C_{0}$-полугруппу $V_{+}(t):=\exp \left\{i A_{0} t\right\}, t \geqslant 0$

b) целая оператор-функция $\left(A_{0}-z I\right)^{-1}$ имеет конечный экспоненциальный тип роста.

Значительный интерес представляет задача описания систем векторов $\left\{f_{k}\right\}_{1}^{n}$, $\left\{g_{k}\right\}_{1}^{n}$, таких, что оператор $A$, связанный с $A_{0} \in \Sigma^{(\exp )}$ равенством $(1)$, генерирует $C_{0}$-полугруппу $U_{+}(t):=\exp \{i A t\}$ или $C_{0}$-полугруппу $U_{-}(t):=\exp \{-i A t\}$, $t \geqslant 0$. В этой заметке мы выделяем класс конечномерных возмущений $A$, удовлетворяющих соотношению (1), в котором сформулированная задача находит свое решение.

Почти всюду положительный $(n \times n)$-матричный вес на вещественной оси будем обозначать через $w^{2}(x), x \in \mathbb{R}$. Далее, через $\mathscr{M}_{n}^{2}$ обозначается класс матричных весов $w^{2}$, удовлетворяющих $\left(A_{2}\right)$-условию Макенхаупта [2]

$$
\sup \left\|\left(M\left(w^{-2}\right)\right)^{1 / 2}\left(M\left(w^{2}\right)\right)^{1 / 2}\right\|<\infty
$$

где $M\left(w^{ \pm 2}\right):=|\Delta|^{-1} \int_{\Delta} w^{ \pm 2}(x) d x, \Delta$ - произвольный интервал на $\mathbb{R}$ и $|\Delta|$ - его длина. По оператору $A_{0} \in \Sigma^{(\exp )}$ и векторам $\left\{g_{k}\right\}_{1}^{n}$ построим $n$-мерную строку

$$
\mathscr{A}_{0}(z, h):=\operatorname{row}\left\{\left(A_{0}\left(A_{0}-z I\right)^{-1} g_{k}, h\right)\right\}_{1}^{n}, \quad h \in \mathfrak{H}, z \in \mathbb{C},
$$

и примем следующее определение.

Определение 1. Пусть операторы $A, A_{0}$ связаны равенством (1). Будем говорить, что $A$ есть $w$-возмущение оператора $A_{0} \in \Sigma^{(\exp )}$ ранга $n$, если существует вес $w^{2}$ класса $\mathscr{M}_{n}^{2}$, такой, что выполняются следующие условия:

1) для каждого $h \in \mathfrak{H}$ функция $\left\|\mathscr{A}_{0}(x, h) w^{-1}(x)\right\|$ принадлежит $L_{2}(\mathbb{R})$;

*Первый автор получал поддержку в рамках госбюджетной темы Украины, номер $0107 \mathrm{U} 000937$. 
2) существует константа $m>0$, такая, что

$$
m\|h\|^{2} \leqslant \int_{\mathbb{R}}\left\|\mathscr{A}_{0}(x, h) w^{-1}(x)\right\|^{2} d x, \quad h \in \mathfrak{H} .
$$

Построим примеры $w$-возмущений операторов $A_{0}$ класса $\Sigma^{(\exp )}$. Пусть $\theta-$ целая внутренняя в верхней полуплоскости $\mathbb{C}_{+} n \times n$-матрица-функция, нормированная условием $\theta(0)=E_{n}([3],[5]$; в работе [5, с. 54] приведено мультипликативное представление матрицы-функции $\theta$ ). Множество таких матриц-функций (м.-ф.) будем обозначать через $J_{n}$. Пусть также $H_{+}^{2}\left(\mathbb{C}^{n}\right)-$ класс Харди $\mathbb{C}^{n}$-значных вектор-функций в полуплоскости $\mathbb{C}_{+} \cdot$ В модельном пространстве

$$
\mathscr{K}_{\theta}:=H_{+}^{2}\left(\mathbb{C}^{n}\right) \ominus \theta H_{+}^{2}\left(\mathbb{C}^{n}\right)
$$

рассмотрим оператор $B$,

$$
B h:=z^{-1}(h(z)-\theta(z) h(0)), \quad h \in \mathscr{K}_{\theta},
$$

который имеет тривиальное ядро, причем $\sigma(B)=\{0\}$ и $\left(B-B^{*}\right) / i \leqslant 0$ (антидиссипативность). Поскольку элементы пространства $\mathscr{K}_{\theta}$ суть целые векторфункции, то оператор $B$ формулой (3) определен корректно. Уместно напомнить ([3, с. 310], [6, с. 91]), что для каждого антидиссипативного вольтеррова оператора $B$ с тривиальным ядром и $n$-мерной мнимой частью существует такая м.-ф. $\theta \in J_{n}$ (характеристическая м.-ф. оператора $B^{*}$ ), что $B$ унитарно эквивалентен оператору вида $(2),(3)$. Далее, для каждого веса $V^{2} \in \mathscr{M}_{n}^{2}$ существует внешняя [3] в $\mathbb{C}_{+}\left(\mathbb{C}_{-}\right)$м.-ф. $V_{+}\left(V_{-}\right)$, такая, что [2]

$$
V^{2}(x) \stackrel{\text { п.в. }}{=} V_{+}^{*}(x+i 0) V_{+}(x+i 0) \stackrel{\text { п.в. }}{=} V_{-}^{*}(x-i 0) V_{-}(x-i 0), \quad x \in \mathbb{R},
$$

где через $V_{+}(x+i 0)\left(V_{-}(x-i 0)\right)$ обозначены некасательные предельные значения м.-ф. $V_{+}(z), z \in \mathbb{C}_{+}\left(V_{-}(z), z \in \mathbb{C}_{-}\right)$на вещественной оси. Обе м.-ф. определяются с точностью до левого постоянного унитарного множителя, и элементы матрицы $V_{+}(z)(z+i)^{-1}\left(V_{-}(z)(z-i)^{-1}\right)$ принадлежат $H_{+}^{2}\left(H_{-}^{2}\right)$.

Рассмотрим в $\mathscr{K}_{\theta}$ систему векторов

$$
G_{k}:=(I+i B)^{-1} \mathbb{P}_{\theta} \frac{V_{+}^{k}(x+i 0)}{x+i}, \quad 1 \leqslant k \leqslant n,
$$

где $V_{+}^{k}(x+i 0)$ обозначает столбец матрицы $V_{+}(x+i 0)$ с номером $k, \mathbb{P}_{\theta}$ - ортопроектор из $H_{+}^{2}\left(\mathbb{C}^{n}\right)$ на подпространство $\mathscr{K}_{\theta}$, а $B$ задается формулой (3). Заметим, что оператор $\widetilde{A}_{0}:=B^{-1}$ принадлежит классу $\Sigma^{(\exp )}$. Пусть $\widetilde{A}-$ плотно заданный в $\mathscr{K}_{\theta}$ оператор, обратный к которому определяется формулой

$$
(\widetilde{A})^{-1} h=\left(\widetilde{A}_{0}\right)^{-1} h+\sum_{k=1}^{n}\left\langle h, F_{k}\right\rangle G_{k}, \quad h \in \mathscr{K}_{\theta},
$$

где $F_{k} \in \mathscr{K}_{\theta}, 1 \leqslant k \leqslant n$, а скобки обозначают скалярное произведение в $\mathscr{K}_{\theta}$. Доказывается, что $\widetilde{A}$ есть $w$-возмущение оператора $\widetilde{A}_{0}$ ранга $n$, причем условия 1), 2) из определения 1 выполняются для веса $w^{2}=V^{2}$. Таким образом, каждая м.фь. $\theta \in J_{n} u$ кажсдый вес $V^{2} \in \mathscr{M}_{n}^{2}$ порождают $w$-возмущения, задаваемые формулами (5), (6).

Обратно, пусть формула (1) задает $w$-возмущение оператора $A_{0}$, которому отвечает вес $w^{2}$ в силу определения 1. Обозначим через $a$ экспоненциальный тип резольвенты $\left(A_{0}-z I\right)^{-1}$. Среди всех левых делителей [3] $\tilde{\theta} \in J_{n}$ м.-ф. $e^{i a z} E_{n}$ су- 
ществуют такие, которые обладают следующим свойством: для каждого $h \in \mathfrak{H}$

$$
\mathscr{A}_{0}(z, h) w_{+}^{-1}(z) \tilde{\theta}(z) \in H_{+}^{2}\left(\mathbb{C}^{n}\right),
$$

где $w_{+}$взята из факторизации вида (4) веса $w^{2}$, а $\mathscr{A}_{0}(z, h)$ имеет тот же смысл, что и в определении 1. В множестве левых делителей $\tilde{\theta}$, для которых имеет место (7), существует «наименьший» $\theta \in J_{n}$ в том смысле, что:

1) каждая м.-ф. $\tilde{\theta}$ содержит $\theta$ в качестве левого делителя;

2) включение (7) имеет место для $\tilde{\theta}=\theta$;

3) $\theta(0)=E_{n}$.

При уже выбранном весе $w^{2} \in \mathscr{M}_{n}^{2}$ внутренняя м.-ф. $\theta$ определяется этими условиями однозначно. Итак, верно обратное утверждение, т. е. каждому w-возмущению отвечает вес $w^{2} \in \mathscr{M}_{n}^{2} u$ м.- $\oint$. $\theta \in J_{n}$. В следующей формулировке $A$, $A_{0}$ связаны равенством (1), оператор $B$ задается формулами (2)-(3), а векторы $G_{k}$ определяются равенствами (5).

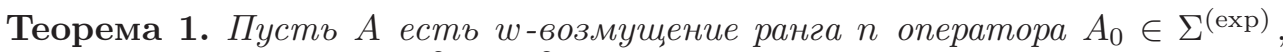
которому отвечает пара $w^{2} \in \mathscr{M}_{n}^{2}, \theta \in J_{n}$. Тогда существует изоморфизм $S$ пространства $\mathfrak{H}$ на модельное пространство $\mathscr{K}_{\theta}$, такой, что

$$
B=S A_{0}^{-1} S^{-1}, \quad S g_{k}=G_{k}, \quad 1 \leqslant k \leqslant n, \quad \widetilde{A}=S A S^{-1},
$$

где $\widetilde{A}$ определяется формулой $(6)$, в которой $F_{k}:=\left(S^{*}\right)^{-1} f_{k}, 1 \leqslant k \leqslant n$.

Из этой теоремы выводится

Следствие 1. Пусть в гилъбертовом пространстве $\mathfrak{H}$ действует оператор $A$, определяемый формулой вида (1), причем $A_{0}^{-1}$ является антидиссипативным оператором с подпространством несамосопряжсенности $\mathscr{L}:=\left(A_{0}^{-1}-\right.$ $\left.\left(A_{0}^{-1}\right)^{*}\right) \mathfrak{H}$ размерности $n$. Если в формуле $(1)$ векторы $g_{k}, 1 \leqslant k \leqslant n$, образуют базис пространства $\mathscr{L}$, то $A$ есть $w$-возмущение оператора $A_{0}$ с постоянным весом $w^{2}(x) \equiv E_{n}, x \in \mathbb{R}$.

2. Описание генераторов $\boldsymbol{C}_{0}$-полугрупп. Каждому оператору $\boldsymbol{A}$, определяемому формулой вида (1), отнесем целую м.-ф. $\Phi(z)$ с элементами

$$
\Phi_{k j}(z)=\delta_{k j}-z\left(A_{0}\left(A_{0}-z I\right)^{-1} g_{j}, f_{k}\right), \quad 1 \leqslant k, j \leqslant n,
$$

и рассмотрим определитель $\Delta(z):=\operatorname{det} \Phi(z)$. Отметим, что $\sigma(A)$ совпадает с множеством $\Lambda:=\left\{\lambda_{k}\right\}$ корней функции $\Delta$.

Пусть $A$ есть $w$-возмущение оператора $A_{0}$, которому в силу рассмотрений разд. 1 отвечает $A_{2}$-вес $w^{2}$ и левый делитель $\theta \in J_{n}$ м.-ф. $e^{i a z} E_{n}$, где $a-$ экспоненциальный тип резольвенты $\left(A_{0}-z I\right)^{-1}$. Заметим, что справедливы неравенства $0<-i \operatorname{Sp}\left(\left.\frac{d}{d z} \theta\right|_{z=0}\right) \leqslant n a$. Через $w_{ \pm}$обозначим внешние м.-ф., которые взяты из факторизации вида (4) веса $w^{2}$.

Теорема 2. Пусть $A$ есть $w$-возмущение оператора $A_{0} \in \Sigma^{(\exp )}$, которому отвечает пара $w^{2}, \theta$. Если при некотором $\varepsilon \geqslant 0$ выполняются условия

1) $\sup _{\lambda_{k} \in \Lambda} \operatorname{Im} \lambda_{k}<\varepsilon$;

2) $\lim \sup _{y \rightarrow+\infty} y^{-1} \log |\Delta(i y)|=-i \operatorname{Sp}\left(\theta^{\prime}(0)\right)$;

3) матричный вес $W_{\varepsilon}^{2}(x):=\Phi(x+i \varepsilon)\left(w_{+}^{*}(x+i \varepsilon) w_{+}(x+i \varepsilon)\right)^{-1} \Phi^{*}(x+i \varepsilon)$ удовлетворяет условию $\left(A_{2}\right)$ на $\mathbb{R}$,

то полугруппа $U_{-}(t):=\exp \{-i A t\}, t \geqslant 0$, принадлежст классу $C_{0}$, причем ее экспоненииальный тип не превосходит $\varepsilon$. 
Обратно, если $U_{-}(t) \in C_{0}$ и тип полугруппь $U_{-}(t)$ равен $\omega$, то при каждом $\varepsilon>\omega$ выполняются условия 1)-2). Кроме того, если при некотором $\delta>0$ м.- $\phi . e^{-i \delta z} \theta(z)$ ограничена в $\mathbb{C}_{+}$, то при любом $\varepsilon>\max \{0, \omega\}$ выполняется условие 3$)$.

Теорема 3. Пусть А есть $w$-возмущение оператора $A_{0}$, которому отвечает пара $w^{2}, \theta$. Если при некотором $\varepsilon \geqslant 0$ выполняются условия

1) $\inf _{\lambda_{k} \in \Lambda} \operatorname{Im} \lambda_{k}>-\varepsilon$;

2) $\lim \sup _{y \rightarrow-\infty}|y|^{-1} \log |\Delta(i y)|=0$;

3) матричный вес $W_{\varepsilon}^{2}(x):=\Phi(x-i \varepsilon)\left(w_{-}^{*}(x-i \varepsilon) w_{-}(x-i \varepsilon)\right)^{-1} \Phi^{*}(x-i \varepsilon)$ удовлетворяет условию $\left(A_{2}\right)$ на $\mathbb{R}$,

то полугруппа $U_{+}(t)=\exp \{i A t\}, t \geqslant 0$ принадлежит классу $C_{0}$ и ее экспоненииальный тип не превосходит $\varepsilon$.

Обратно, если $U_{+}(t) \in C_{0}$ и тип полугруппы $U_{+}(t)$ равен $\omega$, то при каждом $\varepsilon>\omega$ выполняются условия 1)-2). Кроме того, если при некотором $\delta>0$ м.- . $e^{-i \delta z} \theta(z)$ ограничена в $\mathbb{C}_{+}$, то при любом $\varepsilon>\max \{0, \omega\}$ выполняется условие 3$)$.

Следствие 2. Для класса $w$-возмущений из следствия 1 в формулировках теорем 2 и 3 можно положить $w_{ \pm}(z) \equiv E_{n}$.

Остановимся на примере применения теорем 2 и 3 . Пусть $A$ есть $w$-возмущение оператора $A_{0}$ из следствия 1 . Поскольку оператор $L:=A_{0}^{-1}$ антидиссипативен, то существует ортогональный базис $\left\{u_{k}\right\}_{1}^{n}$ подпространства $\mathscr{L}$, такой, что $\left(L^{*}-L\right) h=i \sum_{k=1}^{n}\left(h, u_{k}\right) u_{k}$ для всех $h \in \mathfrak{H}$. Характеристическая м.-ф. оператора $L^{*}$ определяется формулой

$$
\theta_{k j}(z)=\delta_{k j}+i z\left(\left(I-z L^{*}\right)^{-1} u_{k}, u_{j}\right), \quad L=A_{0}^{-1},
$$

и принадлежит классу $J_{n}[5]$. Пусть, кроме того, векторы $\left\{f_{k}\right\}_{1}^{n},\left\{g_{k}\right\}_{1}^{n}$ (см. формулу (1)) образуют базисы подпространства $\mathscr{L}$, т. е. $f_{k}=\sum_{p=1}^{n} d_{k p} u_{p}, g_{k}=$ $\sum_{p=1}^{n} c_{k p} u_{p}$ и матрицы $D:=\left\|d_{k p}\right\|, C:=\left\|c_{k p}\right\|$ обратимы.

Теорема 4. Пусть $A$ есть $w$-возмущение оператора $A_{0}$ из следствия 1, причем обе системы векторов $\left\{f_{k}\right\}_{1}^{n},\left\{g_{k}\right\}_{1}^{n}$ образуют базисы подпространства $\mathscr{L}$, и м.-ф. $\theta$ определяется формулой (8).

Если $\lim _{y \rightarrow+\infty} \theta(x+i y)=0$ равномерно по $x \in \mathbb{R}$, то полугруппа $U_{-}(t)$ принадлежит классу $C_{0}$. Если предположить, кроме того, что матрица $E_{n}+$ $i C D^{*}$ обратима, то классу $C_{0}$ принадлежит также полугруппа $U_{+}(t)$.

Заметим, что в теореме 4 числовой образ оператора $A_{0}^{-1}$ лежит в замкнутой нижней полуплоскости. Из формулы (1) вытекает, что в случае $g_{k}=\sum_{p=1}^{n} Q_{k p} f_{p}$, $1 \leqslant k \leqslant n, \operatorname{Im} Q \leqslant 0\left(Q:=\left\|Q_{k p}\right\|\right)$, возмущение сохраняет это свойство, т. е. $\operatorname{Im}(A f, f) \geqslant 0, f \in \mathscr{D}_{A}$, и, следовательно, полугруппа $U_{+}(t)$ принадлежит классу $C_{0}$. Теорема 4 приводит к такому же выводу, поскольку в этом случае $C=Q D$ и матрица $E_{n}+i Q D D^{*}$ всегда обратима, однако первое условие (о поведении $\theta(x+i y))$ оказывается излишним.

Несмотря на схожесть формулировок обеих теорем, они решают принципиально разные задачи. В теореме 3 речь идет о полугруппе $U_{+}(t)$ при наличии полугруппы $V_{+}(t), t \geqslant 0$, а в теореме 2 дается критерий существования $U_{-}(t)$, в то время как полугруппы $V_{-}(t):=\exp \left\{-i A_{0} t\right\}$ не существует.

Примеры показывают, что условие 3 ) может выполняться и без предположения ограниченности в $\mathbb{C}_{+}$м.ф. $e^{-i \delta z} \theta(z)$. Вместе с тем наша гипотеза за- 
ключается в том, что во всем классе $w$-возмущений от этого предположения отказаться нельзя.

3. Полугруппа сдвигов вдоль периодических в среднем векторфункций. Пусть в пространстве Соболева $W_{2}^{1}\left([0, a], \mathbb{C}^{n}\right)$ задана такая система линейных ограниченных функционалов $\varphi_{k}(1 \leqslant k \leqslant n)$, что никакая их линейная комбинация не продолжается до ограниченного функционала в пространстве $L_{2}\left([0, a], \mathbb{C}^{n}\right)$. Предполагается также, что выполняются условия $\varphi_{k}\left(e_{j}\right)=$ $\delta_{k j}$, где $\left\{e_{k}\right\}_{1}^{n}$ - стандартные орты в $\mathbb{C}^{n}$, рассматриваемые как элемент пространства Соболева. В качестве примера рассмотрим в $L_{2}\left([0, a], \mathbb{C}^{n}\right)$ операторы

$$
A=i \frac{d}{d x}, \quad \mathscr{D}_{A}=\bigcap_{k=1}^{n} \operatorname{ker} \varphi_{k}, \quad A_{0}=i \frac{d}{d x},
$$

причем область определения оператора $A_{0}$ состоит из функций $h \in W_{2}^{1}\left([0, a], \mathbb{C}^{n}\right)$, таких, что $h(0)=0$. Оператор $A$ есть $w$-возмущение оператора $A_{0} \in \Sigma$ ехр ранга $n$, которому отвечает вес $w^{2}(x) \equiv E_{n}$, м.-ф. $\theta(z)=e^{i a z} E_{n}$ и целая м.-ф. $\Phi$ с элементами $\Phi_{k j}(z):=\varphi_{k}\left(e^{-i z a} e_{j}\right)$. Условия 1)-3) теоремы 2 , в которых надо положить $-i S p\left(\theta^{\prime}(0)\right)=n a, w_{+}(z) \equiv E_{n}$, являются необходимыми и достаточными для того, чтобы полугруппа $U_{-}(t)$ принадлежала классу $C_{0}$. На функции $h \in \mathscr{D}_{A}$ полугруппа действует по формуле $\left(U_{-}(t) h\right)(x)=\tilde{h}(t+x)$, где $\tilde{h}-$ единственное продолжение функции $h$ вправо, такое, что $\tilde{h}(t+x) \in \mathscr{D}_{A}$ при каждом $t>0$. Другими словами, для каждого $h \in \mathscr{D}_{A}$ функция $\tilde{h}$ является единственным решением системы

$\varphi_{k}(\tilde{h}(t+x))=0, \quad \tilde{h}(x)=h(x), x \in[0, a], 1 \leqslant k \leqslant n, \quad\|\tilde{h}(a+x)\|_{L_{2}} \leqslant M\|h\|_{L_{2}}$

интегро-дифференциальных уравнений со сдвигом аргумента, удовлетворяющим указанной оценке для норм в $L_{2}\left([0, a], \mathbb{C}^{n}\right)$. Такие продолжения $\tilde{h}$ (по аналогии со случаем $n=1$ [4]) естественно называть периодическими в среднем относительно системы функционалов $\varphi_{k}$. Таким образом, теорема 2 дает критерий разрешимости задачи (9) в терминах м.-ф. $\Phi$.

Эту статью мы посвящаем светлой памяти Марка Григорьевича Крейна; незабываемые годы общения с ним навсегда останутся с нами.

\section{ЛитеРАТУРА}

[1] С. Г. Крейн, Линейные дифференииальные уравнения в банаховом пространстве, Наука, M., 1967. [2] S. Treil, A. Volberg, J. Funct. Anal., 143:2 (1997), 269-308. [3] В. Секефальви-Надь, Ч. Фояш, Гармонический анализ операторов в гильбертовом пространстве, Мир, М., 1970. [4] J. Delsarte, J. Math. Pures Appl., 14 (1935), 403-453. [5] М. С. Бродский, М. С. Лившиц, УМН, 13:1(79) (1958), 3-85. [6] В. А. Золотарев, Аналитические методы спектральных представлений несамосопряжсеных и неунитарных операторов, Из-во Харьковского национального университета им. Каразина, 2003.

Полтавский национальный технический университет, Украина e-mail: gubreev@yandex.ru

University of Missouri-Columbia, USA e-mail: yuri@math.missouri.edu 